\title{
Más allá de los huecos. El tratamiento de los marcadores discursivos en español peninsular mediante las nociones ámbito y posición.
}

\author{
SHIMA SALAMEH JIMÉNEZ \\ Universidad Católica de Valencia \\ shima.salameh@ucv.es
}

\begin{abstract}
Resumen: Presentamos una propuesta didáctica para alumnos de un nivel B2.2 orientada al trabajo de los marcadores discursivos más interactivos en la clase de E/LE. Esta propuesta integra las nociones de posición y ámbito para trabajar con marcadores polifuncionales y conversacionales. Tras una revisión de diferentes materiales didácticos con el fin de detectar el modo en el que se enseñan los marcadores, ofrecemos una secuencia de tres sesiones que toma la idea de (in)corrección política y los debates televisivos como punto de partida.
\end{abstract}

Palabras clave: marcadores discursivos, B2, políticamente correcto, lenguaje hablado

\section{Traducción del título al inglés}

Abstract: This paper presents a didactic exploitation (for B2.2 students) focused on interactive discourse markers in SFL classes. First, a series of Spanish teaching books are reviewed so as to highlight how discourse markers are learned by students. Second, a set of activities is presented. The three sessions of the didactic exploitation are based on the concept politically correct and TV political debates.

Key words: discourse markers, B2, politically correct, spoken language

\section{Introducción ${ }^{1}$}

El tratamiento de los marcadores discursivos requiere herramientas que permitan un análisis homogéneo de sus características (rasgos prosódicos propios, significado procedimental, polifuncionalidad, posibilidad de combinación, etc.). En trabajos recientes (Briz y Pons, 2010) se reivindica la necesidad de incorporar a la descripción de los marcadores dos nociones ${ }^{2}$ : ámbito (es decir, cuál es la parte o componente del discurso a la que afecta) y posición (ocupada con respecto a dicha parte del discurso). $\mathrm{Su}$ empleo es especialmente útil para analizar algunas de las características mencionadas, como la combinatoria de marcadores o su grado de polifuncionalidad, entre otras (Pons, 2018).

La aplicación de estos conceptos en el campo de E/LE también es necesaria, tal y como se ha reivindicado en trabajos recientes (Holgado, 2017; Duque et al., 2019),

\footnotetext{
${ }^{1}$ Este artículo ha sido posible gracias al proyecto de investigación UDEMADIS, Unidades discursivas para una descripción sistemática de los marcadores discursivos en español (FFI-2016-77841-P), financiado por el Ministerio de Economía y Competitividad, AEI y los Fondos FEDER, y por la red de investigación REDT (FFI2017-90738 REDT), financiada también por el Ministerio de Economía y Competitividad y AEI.

${ }^{2}$ Hasta el punto de formar parte de entradas lexicográficas en diferentes diccionarios especializados (Briz, Pons y Portolés, 2008; Fuentes, 2009).
} 
especialmente en los niveles de aprendizaje más altos: el ámbito de los marcadores discursivos permite explicar, por ejemplo, por qué se combinan con un tipo de elementos u otros, o cuál es el significado global que se expresa con dicha combinación (pues mira, pues eso, pero bueno, bueno mira, etc.), un fenómeno propio de la oralidad que se suele trabajar de manera aislada. Por su parte, el estudio de las posiciones de los marcadores permite sistematizar una relación directa con las funciones que expresan, de modo que el alumnado sea capaz de entender el uso de estos elementos sin pasar por memorizar listas de funciones, o hacer actividades basadas en huecos (Salameh Jiménez, 2021).

El presente artículo trata un vacío didáctico en la enseñanza de los marcadores discursivos: la aplicación de las nociones posición y ámbito en la sistematización de su polifuncionalidad. Para ello, se ofrece una propuesta didáctica orientada a la incorporación dichas nociones en el estudio de los marcadores discursivos en grupos de nivel B2.2 en español peninsular (perfectamente adaptable para incorporar usos propios de la variedad atlántica), especialmente aquellos más propios de la oralidad. En primer lugar, presentamos una breve caracterización de los marcadores discursivos y su relación con las nociones ámbito y posición (\$2). Tras una revisión del tratamiento de los marcadores en diferentes materiales $(\$ 3)$, ofrecemos una propuesta desde un enfoque comunicativo que incorpore estos conceptos (§4), siempre en línea con los requisitos y objetivos de aprendizaje del MCER y el PCIC, y con una integración completa de los marcadores discursivos modales e interpersonales.

\section{Marcadores discursivos: polifuncionalidad, ámbito y posición}

Los marcadores discursivos son elementos de significado procedimental que contribuyen a que los procesos de comunicación sean exitosos, puesto que proporcionan a hablantes/oyentes/lectores instrucciones para asimilar la información o producirla (Portolés, 2001). Los estudios generales sobre marcadores discursivos (Schourup, 1999, Fischer, 2006; Portolés, 1993; Cuenca, 2006; Loureda y Acín, 2010; Crible, 2018) suelen definirlos en función de sus diferentes clasificaciones, categorías, denominaciones ${ }^{3}$ o rasgos. A pesar de presentar algunas diferencias en cuanto a su concepción (Fischer, 2006), los autores coinciden en remarcar:

a. Su carácter polifuncional (Schiffrin, 2015: 62; Bazzanella, 1986), independientemente del enfoque desde el que se aborde (homonimia, Jucker, 1993; monosemia, Fretheim, 2000; y polisemia, Fischer, 2006; Hansen, 2008);

b. Su alto grado de movilidad en diferentes posiciones discursivas. Habitualmente, se habla de posición inicial, final, medial, o incluso independiente (Martín Zorraquino y Portolés, 1999; Fraser, 1999; Tanghe, 2016); algunos trabajos abordan las diferentes posiciones mediante los conceptos de periferia izquierda y derecha (Traugott, 2014; Degand, 2014; Salameh, Estellés y Pons, 2018);

\footnotetext{
3 Otras denominaciones relacionadas con marcador discursivo son marcador pragmático, partícula discursiva, operador, conector, partícula modal, etc. No existe una equivalencia directa entre todas ellas, sino que algunas son más generales y otras más específicas.
} 
c. Su ámbito variable, al afectar a segmentos discursivos más reducidos (como sintagmas o enunciados) o más amplios (intervenciones, secuencias textuales, o textos completos) (Cuenca, 2006; Briz y Pons, 2010), e incluso combinándose con otros marcadores discursivos (Fraser, 2015; Pons, 2018).

Por tanto, polifuncionalidad, posición y ámbito son nociones que determinan el análisis de los marcadores discursivos en cualquier lengua: por ejemplo, la polifuncionalidad de los marcadores plantea en muchas ocasiones análisis que no muestran un límite claro sobre cuál es la función predominante y los valores secundarios que han desarrollado, si existe o no una interrelación entre ellos, o si la posición o zona discursiva y el ámbito en los que aparecen condicionan posibles diferencias de uso en contextos orales o escritos (Montañez Mesas, 2015). Asimismo, el ámbito también influye en la presencia de unos valores $\mathrm{u}$ otros en diferentes contextos (como cuando bueno en español introduce una intervención como respuesta a otro hablante refleja un valor interpersonal, distinto a cuando introduce un fragmento de información más pequeño y permite matizar una idea). Obsérvense los casos (1) a (3):

(1) Vendrás a ver el partido del sábado, ¿no?

(2) Esta chica estuvo hablándome de muchas cosas interesantes / ¿no? Lo que pasa es que yo no estaba centrada, ehh, ¿no? Porque el examen no me salió bien y-y

(3) A: Creo que no iremos de vacaciones juntos

B: Pues bueno / qué le vamos a hacer...

En los contextos (1) y (2), se utiliza el marcador discursivo ¿no? con dos valores distintos: buscar una confirmación por parte de otro hablante (1), y apoyar la formulación del mensaje mientras se emite (2). Aparentemente, podría pensarse que en ambos casos el marcador aparece en posición final de un contenido previo, pero existe una diferencia entre ellos: mientras en (1) el hablante cierra su intervención y la proyecta hacia la de otro hablante mediante el marcador, en (2) le permite seguir formulando la intervención y añadir más información, por lo que la proyección del marcador es más acotada, interna. Esta diferencia de posición y ámbito refleja una diferencia funcional, por lo que es posible afirmar que casos como (1) tienden a ser interpersonales, y casos como (2), apoyos a la formulación. Por su parte, en (3) se observa que los marcadores pues y bueno no funcionan aisladamente, sino como un conjunto al que, además, se le puede atribuir un significado: desacuerdo parcial (B acepta lo que dice A, pero no lo comparte).

Estas ideas, de corte teórico, son extrapolables a la enseñanza de los marcadores del español a extranjeros (ver, entre otros, Duque et al., 20194; Martínez Sánchez, 2011; Holgado Lage, 2017): es habitual que muchos estudiantes que ya tienen un dominio alto del idioma, gracias a su intuición lingüística o por paralelismos con su lengua materna, puedan detectar que ciertos marcadores se utilizan en unas posiciones y no en otras, o que se relacionen con un tipo de unidades. Sin embargo, no todo el alumnado tiene por qué responder de este modo al estudiar las características de los marcadores discursivos. $\mathrm{Si}$, además, dicho estudio se plantea sin explicitar una relación sistemática entre

\footnotetext{
${ }^{4}$ Los autores analizan el comportamiento, en relación con las posiciones, de marcadores como o sea, es decir vamos o vaya, especialmente en contextos coloquiales (Duque et al., 2019: 83).
} 
polifuncionalidad, ámbito y posición, su aprendizaje puede perder significatividad y convertirse en un proceso automático basado en listas funcionales y actividades enfocadas a completar y producir textos, sin un trabajo con contextos de uso reales.

Como consecuencia, un enfoque más textual vincula el estudio de los marcadores discursivos con contextos escritos y más formales, así como con una mayor especialización en marcadores textuales, sin prestar tanta atención a los modales e interpersonales, más utilizados en contextos orales (contrariamente a lo que se recomienda desde el PCIC o el MCER). Esto puede observarse en el tratamiento de los marcadores que, desde los primeros niveles, brindan algunos manuales de E/LE, analizados a continuación.

\section{Marcadores discursivos y su tratamiento en manuales de español}

\subsection{PCIC y MCER: claves teóricas}

Los marcadores discursivos, como indica Muñoz Pérez (2020), formarían parte de la cohesión en los contenidos curriculares del PCIC, y se encuentran en los inventarios de Gramática (2.3., 2.4., 2.7., 2.8., 2.14., 2.15.) y tácticas y estrategias pragmáticas (6.1.1., 6.1.2., y 6.1.4.) (Muñoz Pérez, 2020: 150). Según el PCIC, es recomendable abordar el aprendizaje de los marcadores discursivos ya desde niveles iniciales (A1-A2). Desde entonces, los usos textuales (conectores) e interpersonales (controladores del contacto) amplían la nómina de tipos progresivamente para incorporar los estructuradores de la información (a partir de A2), los marcadores de reformulación, y los operadores discursivos (a partir de B1). A medida que se incrementa el nivel de lengua, el grado de detalle y la cantidad de marcadores incluidos incrementa (sobre todo los más textuales), pero las tipologías siempre son las mismas (C1-C2). Esta idea de progresión también se desprende del MCER, aunque con foco en los conectores de lo escrito, cuyo aprendizaje va de lo más sencillo (cohesión de ideas mediante conectores como $\mathrm{y}$, también, etc.) hasta procesos más complejos y variados a partir de un nivel B2 (MCER, 2002: 37).

\subsection{Los marcadores en los manuales de español: algunos problemas}

Según el PCIC y el MCER, es esperable, pues, que los materiales de enseñanza del español trabajen los marcadores discursivos desde etapas tempranas, y con un mayor desarrollo a partir de niveles intermedios. Para comprobar qué tratamiento reciben, hemos analizado ocho manuales actuales pertenecientes a diferentes niveles (A1-C1) y editoriales, con el fin de obtener una muestra cualitativa representativa ${ }^{5}$. Estos materiales han sido seleccionados por su relevancia en el panorama de enseñanza de E/LE actual, así como por ser bastante completos en cuanto a los contenidos en todos los niveles y competencias.

\footnotetext{
5 Por cuestiones de extensión, se ha excluido de la cala el manual Aula Internacional 5 (para nivel B2.2), puesto que se analizan en el artículo otros materiales pertenecientes a este nivel (como, por ejemplo, Abanico).
} 


\begin{tabular}{|l|} 
Aula Internacional 1, 2, 3, $4(\mathrm{~A} 1, \mathrm{~A} 2, \mathrm{~B} 1, \mathrm{~B} 2.1)$ \\
\hline Pasaporte B1 y B2 \\
\hline Abanico B2 \\
\hline C de C1 \\
\hline
\end{tabular}

Tabla 1. Materiales empleados para el análisis de los marcadores discursivos en manuales de E/LE

Tras su revisión, podemos afirmar que estos manuales abordan los marcadores discursivos desde etapas iniciales, y buscan ir en la misma línea que el PCIC o el MCER, aunque es cierto que parece existir un mayor peso de lo escrito que de lo oral (y, por tanto, de la comprensión lectora y escrita, o la producción escrita en cuanto a las competencias), y que puede darse alguna carencia en el estudio de estos elementos. Asimismo, destaca la falta de mención a las nociones de posición y ámbito, o a la polifuncionalidad.

\subsubsection{A1 - A2}

Aula Internacional 1 (A1) introduce elementos como y/ni/también/tampoco, clasificados como recursos gramaticales y analizados al final del material como conectores. Sin embargo, no hay presencia de marcadores interactivos o controladores del contacto (sobre todo los de modalidad interrogativa, como ¿no? o ¿eh?) para pedir confirmación u opinión (PCIC, $\S 1.4, \S 2.1$.), que también pueden trabajarse, de manera adaptada, en etapas iniciales debido a su alta frecuencia de uso en español oral (coloquial prototípico y periférico -entrevistas, debates, etc.-, Briz, 2011). Con respecto a la forma de aprender estos elementos, puede observarse que, muy inductivamente, los estudiantes tienen acceso a usos orales de también o tampoco, trabajados previamente por escrito, o a la adición de información mediante $y$ en contextos dialógicos (Aula Internacional 1, págs. 76-78), aunque sin pautas explícitas para su uso.

\begin{tabular}{|c|c|c|c|c|c|c|}
\hline INGLÉS & FuTBOL & $\mid$\begin{tabular}{l|l|} 
INGLES & YOGA \\
FERNANDO FERNANDO
\end{tabular} & $\begin{array}{l}\text { CENA } \\
\text { CONN } \\
\text { CARMEN }\end{array}$ & $\sum^{1 E N \mid>}$ & $\begin{array}{l}D E L A \\
A B \cup E A\end{array}$ & $\begin{array}{l}\text { C. ¿Tienes algo en común con } \\
\text { Pedro? Escríbelo y coméntalo } \\
\text { con tus compañeros. }\end{array}$ \\
\hline $\begin{array}{l}\text { - depor } \\
\text { - famili }\end{array}$ & & $\begin{array}{l}\text { - maniático } \\
\text { - perezoso }\end{array}$ & & $\begin{array}{l}\text { yanizado } \\
0\end{array}$ & & $\begin{array}{l}\text { Yo tambien hage deporte casi } \\
\text { todos los dias. }\end{array}$ \\
\hline
\end{tabular}

\section{SIEMPRE HAGO LA CAMA DESPUÉS DE DESAYUNAR}

En parejas. Imaginad que un amigo os dice estas frases. ¿Qué le respondéis? Habladle de vuestras costumbres.

1. Me lavo los dientes antes y después de comer.

2. No voy casi nunca al gimnasio antes de trabajar

3. A veces estudio por la noche, después de cenar.

4. Nunca veo la televisión después de cenar

yo si / yo no / yo también / yo tampoco

yo siempre / yo nunca / yo a veces

5. Me ducho siempre antes de acostarme.

yo antes / yo después

Imagen 1. Aula Internacional 1. Tratamiento de tampoco y también en contextos dialógicos, para introducir respuestas y marcar acuerdo/desacuerdo al hablar sobre rutinas diarias. 


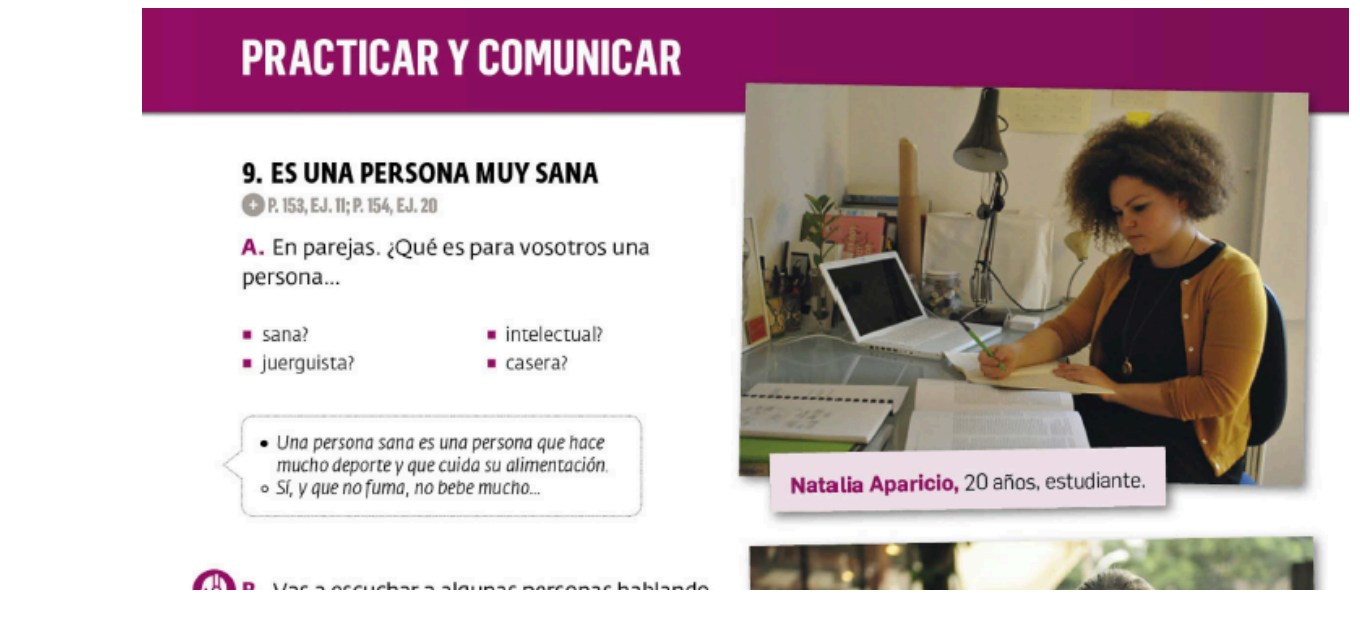

Imagen 2. Aula Internacional 1. Tratamiento de y en contextos dialógicos, para introducir respuestas y añadir información a lo que dice otro hablante.

En ninguno de los casos se consideran aspectos como la posición o el ámbito (por ejemplo, sería útil resaltar que muchos de estos usos de tampoco y también aparecen al principio de una intervención para introducir reacciones de diferente tipo, o que $y$ no se comporta igual cuando relaciona palabras que cuando introduce respuestas).

Con respecto a Aula Internacional 2 (para A2), destaca el hecho de que no mantiene esta introducción al uso de conectores: no hay un tratamiento explícito de las tipologías establecidas por el PCIC, a pesar de que es ya posible que los estudiantes conozcan algunos marcadores consecutivos (como por eso, entonces) o controladores del contacto (sobre todo de base imperativa, como oye/oiga, mira/mire, etc.), con función fática y previos a una explicación (PCIC, §9.3).

\subsubsection{B1 - B2}

Los materiales de nivel intermedio (B1 y B2) reflejan una mayor presencia de marcadores discursivos, aunque Pasaporte B1 y B2 no ofrecen un tratamiento directo, a diferencia de Aula Internacional (B1, B2.1.) y Abanico B2, razón por la que no se describen a continuación. En Aula Internacional 3 (B1) se trabaja con conectores textuales para establecer relaciones de causa-consecuencia (como, porque, así que, de modo que, etc.) y concesión y oposición (pero, sin embargo, aunque, sino). Aunque hay algunos apuntes sobre usos orales de algunos de estos conectores (pero si se puede utilizar en la lengua oral para presentar las razones de nuestro desacuerdo, p. 233), este material se aplica sobre todo a contextos escritos, a través de la comprensión y la producción, y con un nivel de expresión más formal: 

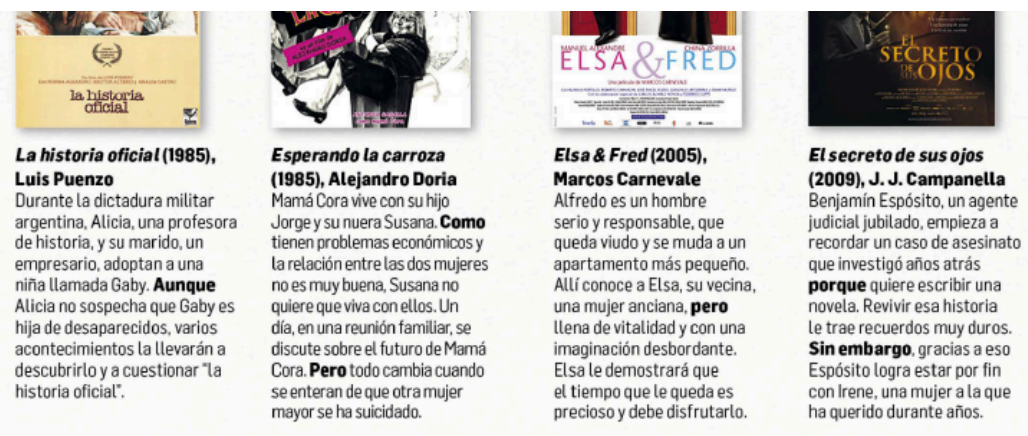

Imagen 3. Aula Internacional 3. Conectores de oposición en contextos escritos.

\section{6. ¿DE QUE VA? $\odot P .199, E J .6 ;$ P. 200, EJ.7}

A. Lee los mensajes de móvil que algunos viajeros han enviado a sus amigos y familiares. Relaciónalos con las fotos de los lugares que aparecen debajo.

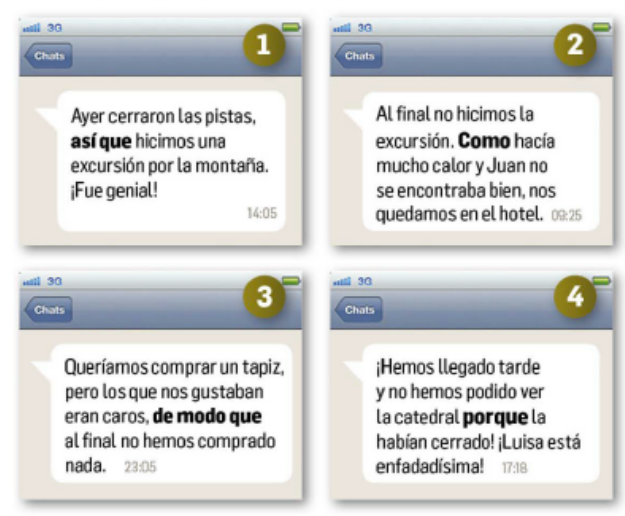

B. Fijate en los conectores marcados en negrita. ¿Cuáles sirven para presentar una causa? ¿Cuáles sirven para presentar una consecuencia?

c. Piensa en un viaje que hiciste. Escribe cuatro mensajes contando experiencias que viviste. En cada mensaje tienes que usar uno de los conectores del apartado $\mathrm{A}$.

noventa y nueve | 99

Imagen 4. Aula Internacional 3. Conectores de causa-consecuencia en contextos escritos.

De nuevo, en la información teórica proporcionada a los alumnos (imagen 5) no aparecen las nociones de posición y ámbito con respecto a estos conectores, que suelen utilizarse en posición medial y muy integrados en el fragmento (es decir, tienen un ámbito más reducido, en ocasiones funcionan como conectores de dos lugares). Únicamente se hace mención al orden de aparición de causas y consecuencias, o a que el segundo elemento de una oposición anula al primero. Se introduce una función y un ejemplo para cada caso. 


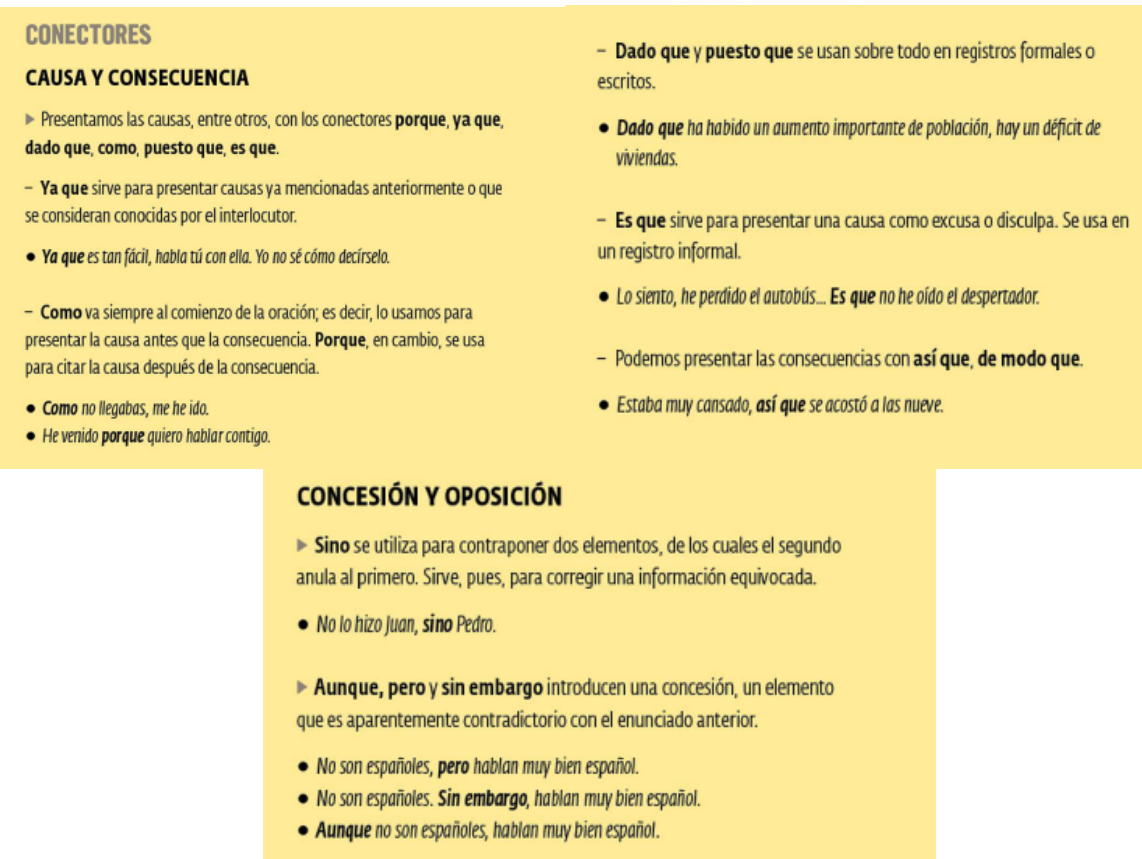

Imagen 5. Aula Internacional 3. Base teórica conectores de causa-consecuencia y concesión-oposición.

La información y los contenidos del manual coinciden con los recomendados por el PCIC (§1.2.), sobre todo en relación con los procesos de justificación y contraargumentación (§1.4.1., §2.3.). No obstante, los marcadores interactivos (¿no?, ¿sabes?) se trabajan solo de manera indirecta, como recursos de la oralidad en una actividad dialógica (aunque a partir de un texto leído al que reaccionar) relacionada con los tiempos verbales en las anécdotas (imagen 6). No se proporcionan pautas descriptivas para la actividad, y tampoco acerca la polifuncionalidad de estos elementos, o de la posibilidad de que aparezcan en diferentes posiciones discursivas.

\section{A Mİ, UNA VEZ...}

En grupos de tres, cada uno elige una anécdota y se la cuenta a sus compañeros con la ayuda de estos recursos. Los otros escuchan y reaccionan. Podéis grabaros.

\begin{tabular}{|c|c|c|c|c|c|c|c|c|c|}
\hline porque & resulta que & y entonces & total, que & asíque & ¿no? & ¿Sabes? & al final & como & de modo qué \\
\hline \multicolumn{3}{|c|}{$\begin{array}{l}\text { Hace un tiempo / En un parque } \\
\text { / Ver a una chica y a un chico } \\
\text { peleándose / La chica estar muy } \\
\text { asustada / Llamar a la policía / } \\
\text { Estar rodando una película }\end{array}$} & \multicolumn{3}{|c|}{$\begin{array}{l}\text { El otro día / Llevar solo G euros } \\
\text { / Decidir comprar un billete de } \\
\text { lotería / Ganar un premio de } 600 \\
\text { euros / Invitar a unos amigosa } \\
\text { una barbacoa / Pasarlo muy bien }\end{array}$} & \multicolumn{4}{|c|}{$\begin{array}{l}\text { Una vez / Encontrar a alguien en un tren } \\
\text { / Empezar a hablarle y preguntarle } \\
\text { por su vida / Pensar que lo conocía / La } \\
\text { otra persona mirarme con cara rara / } \\
\text { Parecerse mucho a un amigo }\end{array}$} \\
\hline
\end{tabular}

Imagen 6. Aula Internacional 3. Actividad en la que aparecen marcadores de contacto en nivel B1.

Por su parte, Aula Internacional 4 (B2) incluye más conectores y una nómina más amplia con respecto al nivel anterior: conectores para matizar, añadir, contraponer y desmentir información y ordenadores de la información -aunque se clasifican como recursos gramaticales-, así como conectores para argumentar con construcciones del tipo en mi opinión, tal como lo veo yo, estoy convencido de, para mí, etc. Nuevamente, las actividades se plantean desde un enfoque muy textual, con desarrollo de competencias lectora y escrita ( $\mathrm{o}$, a lo sumo, auditiva), sin atender a los usos más orales, o, simplemente, a marcadores de esas mismas tipologías que puedan utilizarse en 
contextos conversacionales (pero, es que, etc., o combinaciones de marcadores, i.e. pero es que, etc.) (imágenes 7 a 9).

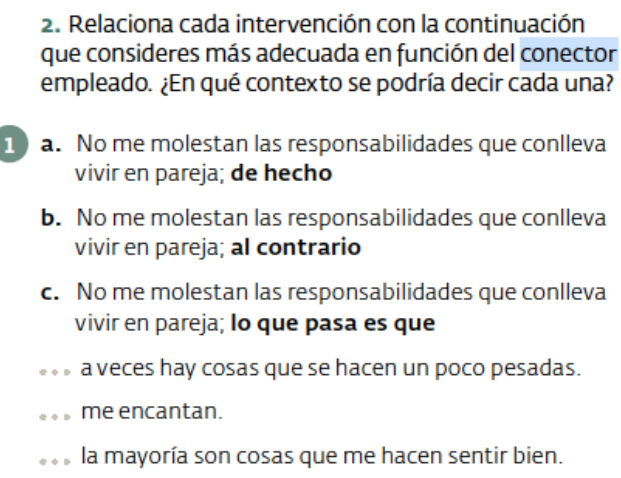

b. No me molestan las responsabilidades que conlleva vivir en pareja; al contrario

c. No me molestan las responsabilidades que conlleva vivir en pareja; lo que pasa es que

... a veces hay cosas que se hacen un poco pesadas.

... me encantan

... la mayoría son cosas que me hacen sentir bien.

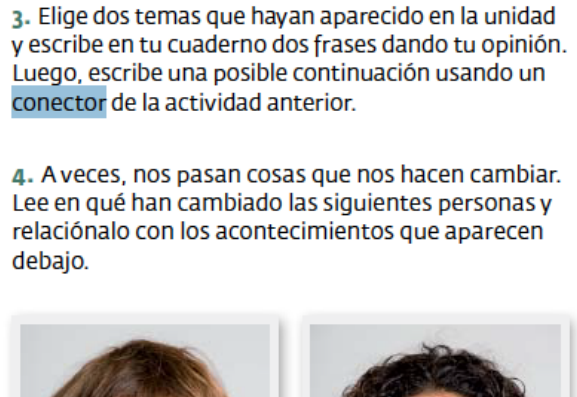

Imagen 7. Aula Internacional 4. Actividades para trabajar conectores en contextos de matización y oposición de ideas.

\section{NO ES OUE ME PROHÍBA ENAMORARME, LO QUE PASA ES OQUE... $\odot$ P. 110, EJ.2-3; P. 114, EJ. 14}

A. Subraya en el texto de la página 53 los siguientes conectores.

En tu cuaderno, intenta traducir a tu idioma las frases en las que aparecen.

\begin{tabular}{|c|c|c|c|}
\hline $\begin{array}{l}\text { Para matizar } \\
\text { una información }\end{array}$ & $\begin{array}{l}\text { En realidad, } \\
\text { De hecho, }\end{array}$ & $\begin{array}{l}\text { Para desmentir una } \\
\text { información dando } \\
\text { una explicación }\end{array}$ & $\begin{array}{l}\text { No es que... } \\
\text { Lo que pasa es que... }\end{array}$ \\
\hline $\begin{array}{l}\text { Para organizar la } \\
\text { información y añadir } \\
\text { nuevos elementos }\end{array}$ & $\begin{array}{l}\text { Además, } \\
\text { Por otro lado, }\end{array}$ & $\begin{array}{l}\text { Para oponer } \\
\text { información }\end{array}$ & Al contrario, \\
\hline
\end{tabular}

B. Escribe tres frases dando tu opinión sobre este tema: ¿Vivir solo o en pareja?

En cada frase tienes que usar un conector del apartado anterior.

Imagen 8. Aula Internacional 4. Actividades para trabajar conectores en contextos de adición, justificación u oposición. 


\section{POR UNA PARTE...}

A. En parejas, intentad completar con conectores estos fragmentos de la entrevista con el experto en turismo. Luego, comparad vuestras respuestas con las de otra pareja.

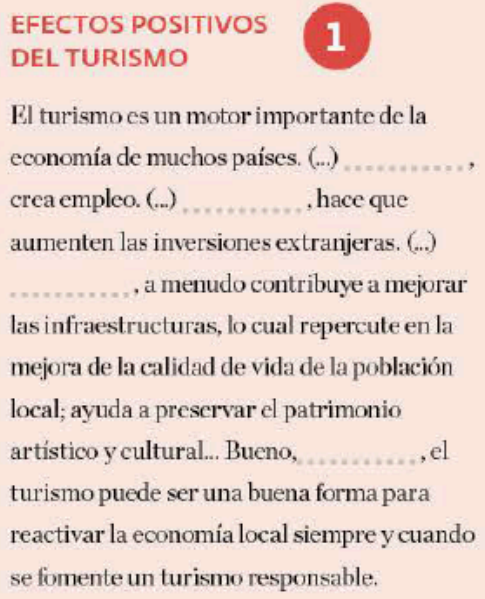

El turismoes un motor importante de la

Imagen 9. Aula Internacional 4. Actividades para trabajar con ordenadores de la información a partir de grabaciones de audio y escritura.

En cuanto a la relación con los contenidos del PCIC, sí es directa y explícita: se trabajan conectores aditivos (§1.2.1., §14.1), consecutivos (§15.3.7.), justificativos (§15.3.4.), contraargumentativos (§15.3.9.), estructuradores de la información (§1.2.2.), y algunos recursos de refuerzo argumentativo (§1.2.4.). Sin embargo, su tratamiento teórico resume las formas empleadas para cada caso sin incidir en el peso de las nociones posición y ámbito, aunque sea de modo adaptado.

Abanico (B2) se centra en el uso de algunos marcadores textuales en actividades que fomentan las competencias escritora y lectora, aunque como parte de actividades más amplias, centradas en aprender a relatar o sobre la comprensión de textos (imágenes 10 y 11). La nómina de ejercicios y materiales sobre marcadores es menor que en Aula Internacional.

\section{3 Que pasó con Blena?}

En parejas, poneos en el lugar de Pepe Luis y reconstruid su pequeña historia con Elena. Deberéis decidir, para cada verbo en negrita, imperfecto o indefinido, de acuerdo con vuestra interpretación de los acontecimientos, y no podéis cambiar el orden. Las fórmulas del cuadro a la derecha os ayudarán a cohesionar el texto.

\begin{tabular}{|c|c|}
\hline $\begin{array}{l}\text { ¿QUÉ PASÓ AYER? } \\
\text { 1. Ser un dia triste } \\
\text { 2. Estar pensando qué hacer con Elena (yo) } \\
\text { 3. Bajar a la calle (yo) } \\
\text { 4. Ir al bar "Manolo" (yo) } \\
\text { 5. Tomarme una cerveza (yo) } \\
\text { 6. ONidarme de Elena (yo) } \\
\text { 7. Acordarme otra vez de Elena (yo) } \\
\text { 8. Estar Iloviendo } \\
\text { 9. Volver a mi casa (yo) } \\
\text { 10. Encontrarme a Elena en ropa interior (yo) } \\
\text { 11. No querer hablar conmigo (ella) } \\
\text { 12. Explicarle mi relación con Ana (yo) } \\
\text { 13. Irse (ella) } \\
\text { 14. Decirr "Te quiero" (yo) } \\
\text { 15. Yolver sonriendo (ellia) }\end{array}$ & $\begin{array}{l}\text { Pues } \\
\text { Pero.. } \\
\text { Porque } \\
\text { Y... } \\
\text { Cuando....... } \\
\text { De pronto,... } \\
\text { Mientras....... } \\
\text { De repente, .... } \\
\text { Entences.... } \\
\text { Así que..... } \\
\text { Despues... } \\
\text { Yluega.... } \\
\text { En ese momento... } \\
\text { Al final, ... } \\
\text {...un rato... } \\
\text {....diez minutos... }\end{array}$ \\
\hline
\end{tabular}


Imagen 10. Abanico. B2. Actividades para trabajar el relato en relación con una historia ya dada. Se incluyen algunos marcadores discursivos.

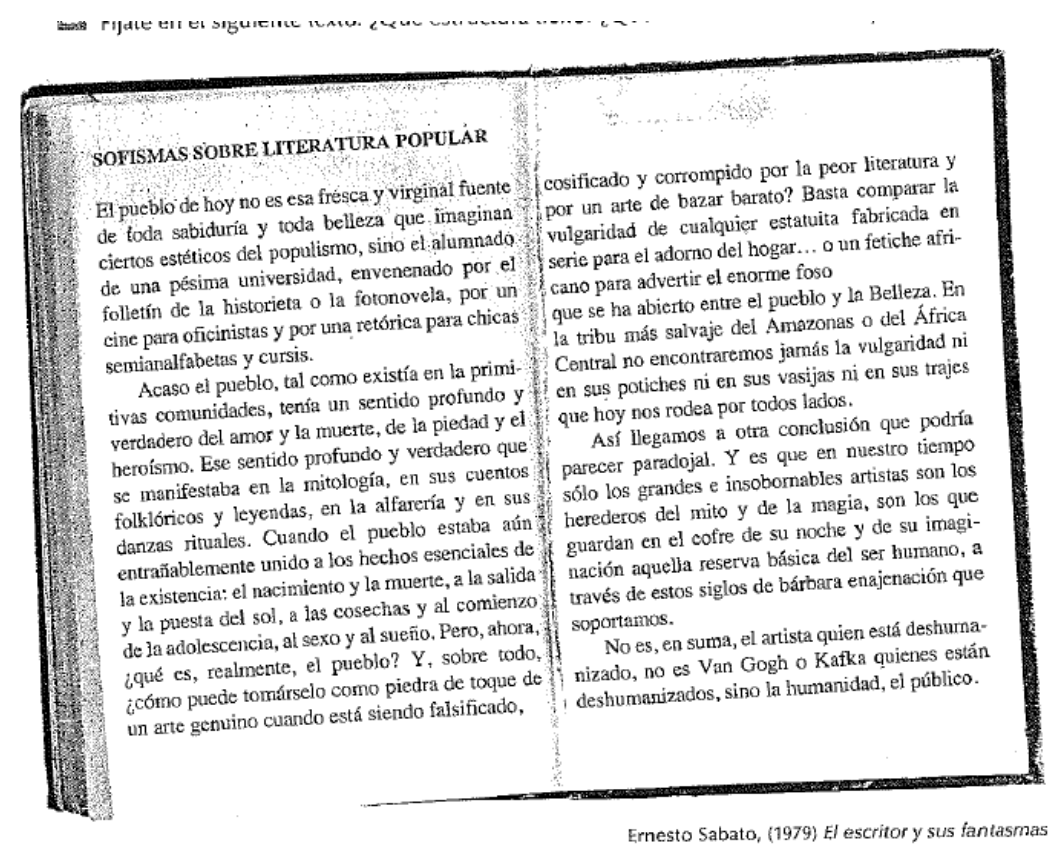

Imagen 11. Abanico. B2. Taller de escritura para trabajar la estructura de los textos y los párrafos.

Texto de Ernesto Sábato con marcadores discursivos textuales y construcciones formales.

Si bien es cierto que los estudiantes pueden partir inductivamente de la muestra para trabajar con los marcadores empleados, y que este es un modo efectivo de hacerlo, es necesario brindarles más herramientas para su correcto uso. Por último, en Abanico tampoco se incluyen marcadores modales o interactivos, contextos orales, ni un análisis detallado de la polifuncionalidad de estos elementos y su vínculo con la posición y el ámbito.

\subsubsection{C1}

El manual $\mathrm{C}$ de $\mathrm{C} 1$ es uno de los más orientados al tratamiento de la oralidad en el aula de E/LE, sin por ello renunciar al estudio de lo textual. Con un tono onomasiológico (es decir, partir de la función/proceso/fenómeno para llegar a las formas/construcciones que permiten expresarlos), los recursos didácticos se articulan en torno a la relación entre teoría y actividades, con una amplia batería de recursos aplicables en contextos más o menos formales, conversacionalmente y por escrito. En concreto, el trabajo con los conectores es más explicito que en otros manuales: normalmente, se parte de una muestra que permite extraer reglas y funciones para que el alumnado pueda aprenderlos ( $\sin$ tener que ir a una sección final de teoría) y, seguidamente, aplicarlos en otras actividades. Aunque hay algunos ejercicios con huecos, forman parte de una actividad más amplia, que busca un aprendizaje significativo de los marcadores. 


\section{TRABAJAR LA GRAMATICA}

\section{CONDICIONES}

B.7 Lee estos fragmentos del contrato y observa las expresiones destacadas. Todas introducen una condición, pero aportan matices diferentes. Escribe en el cuadro qué estructura corresponde a cada uso.

\begin{tabular}{|c|c|c|}
\hline $\begin{array}{l}\text { "En el caso de que no se } \\
\text { proceda al pago del precio total } \\
\text { del viaje en las condiciones } \\
\text { señaladas, se entenderá que el } \\
\text { consumidor desiste del viaje } \\
\text { solicitado." }\end{array}$ & $\begin{array}{l}\text { "La información contenida en el } \\
\text { programa/oferta es vinculante } \\
\text { para el organizador o detallista, } \\
\text { salvo que concurra alguna de } \\
\text { las siguientes circunstancias." }\end{array}$ & $\begin{array}{l}\text { rio tendrá derecho al reembolso del } \\
\text { precio siempre y cuando la agencia se } \\
\text { otificado por escrito con un mínimo de } \\
\text { de antelación." } \\
\text { 6ram, p. 181-182>18 }\end{array}$ \\
\hline \multicolumn{3}{|l|}{ Conectores condicionales } \\
\hline $\begin{array}{l}\text { a. } \rightarrow \text { Introduce una condición. } \\
\text { Corresponde a si en un registro formal. } \\
\text { b. } \rightarrow \text { introduce un requisito } \\
\text { para que algo se cumpla. Corresponde a } \\
\text { a condición de (que). }\end{array}$ & \multirow{2}{*}{$\begin{array}{l}\text { Otras estructuras establecen la misma } \\
\text { relación lógica: } \\
\text { a. Introducir una condición: de + infinitivo. } \\
\text { b. Introducir un requisito: siempre que, } \\
\text { solo si, con tal de que (coloq.), cuando. } \\
\text { c. Introducir una excepción: a menos que, } \\
\text { a no ser que, excepto si/que. }\end{array}$} & \multirow{2}{*}{$\begin{array}{l}\text { Todas las expresiones para poner con- } \\
\text { diciones se construyen con subjuntivo, } \\
\text { excepto las que incluyen la forma si, } \\
\text { que se construyen como las condicionales } \\
\text { con si, y de + infinitivo. } \\
\text { En el caso de acepta modificaciones } \\
\text { según el grado de probabilidad: } \\
\text { en el hipotético caso de que.. }\end{array}$} \\
\hline $\begin{array}{l}\text { c. } \rightarrow \text { Introduce una excep- } \\
\text { ción. Corresponde a con la excepción } \\
\text { de que. }\end{array}$ & & \\
\hline
\end{tabular}

Imagen 12. C de C1. Muestra de trabajo con conectores condicionales. A partir de una muestra real, se extraen funciones y tipos para que el alumnado los aprenda. Incluye ejercicios complementarios.

Aunque, en su mayoría, las actividades son de corte inductivo, puesto que están orientadas a un nivel de idioma alto, y sus usuarios ya conocen muchos de los tipos de marcadores discursivos de estudio recomendados por el PCIC.

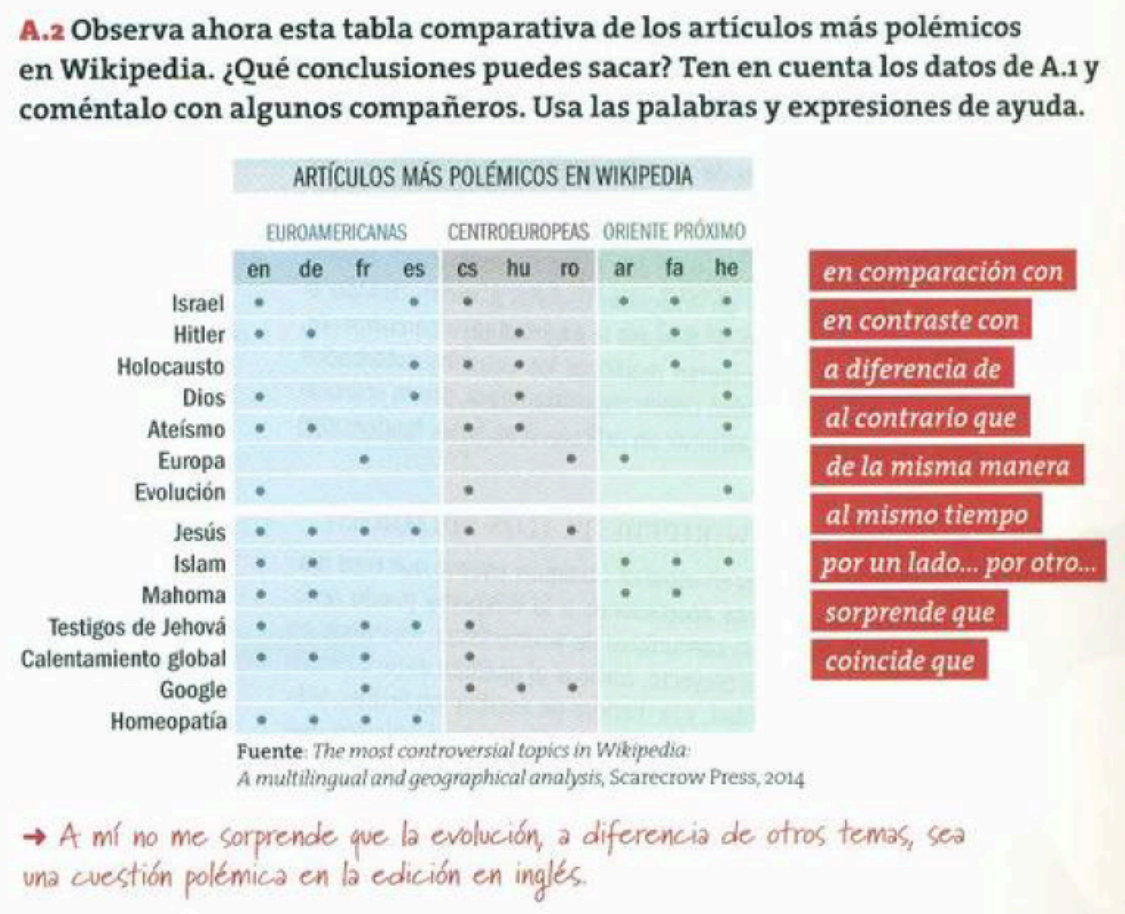

Imagen 13. C de C1. Actividad a partir de un artículo para comentar dialógicamente los resultados del mismo. Utilizan, entre otros recursos, conectores de contraste, ordenadores de la información, etc. 
$\mathrm{C}$ de $\mathrm{C} 1$ incluye, principalmente, conectores condicionales, argumentativos (§1.2.1.), consecutivos (\$15.3.7), estructuradores de la información (§1.2.2.), y operadores discursivos o marcadores de refuerzo (§1.2.4.), siempre a partir de la observación del discurso y de muestras reales (textos, páginas web, artículos de prensa, e incluso algunas muestras audiovisuales). Esto coincide con la recomendación del PCIC, a excepción de la parte correspondiente a los marcadores de reformulación, que no se tratan (para el problema del tratamiento de la reformulación y sus marcadores, ver Salameh, 2020), aunque también se podría incidir más en el uso de marcadores de la oralidad o, directamente, en un tratamiento más explícito de los marcadores en general.

El aspecto más destacable del manual es que sí hace referencia a los conceptos de posición y ámbito (imagen 14) en las fichas de gramática (puede ocupar la posición inicial, intermedia o final de la oración; separación del resto de elementos mediante pausas o comas, etc.). Este hecho facilita su tratamiento por parte del profesor, que puede aprovechar la información para brindársela al alumnado, así como incidir directamente en este rasgo para describir la polifuncionalidad de los marcadores analizados. El tipo de explicación, además, es bastante accesible para los estudiantes.

\footnotetext{
cuando los sujetos del verbo principal y el verbo de la oración final coinciden. Usamos que + subjuntivo cuando los sujetos de ambas oraciones son diferentes.

- Fuimos a Barcelona para conocer a nuestro nuevo sobrino. ,el sujeto es nosotros

- He hecho comida de más para que puedas llevarte mañana al

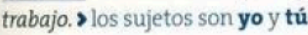

La estructura $\langle$ nexo + que + subjuntivo $\rangle$ admite dos tiempos verbales: el presente y el imperfecto de subjuntivo. Usamos el presente para hablar de situaciones que son posteriores al momento de hablar (normalmente cuando el verbo de la oración principal está en presente, pretérito perfecto o futuro).

- Hago siempre comida de más para que puedas llevarte al trabajo. - He hecho comida de más para que puedas llevarte mañana al trabajo.

- Mañana haré comida de más para que puedas llevarte al trabajo.

En cambio, usamos el imperfecto de subjuntivo para hablar de situaciones que son posteriores a un momento del pasado (normalmente cuando el verbo de la oración principal está en un tiempo de pasado o condicional)

- Ayer hice comida de más para que pudieras llevarte al trabajo.

- Si tuviera tiempo, haria comida de más para que pudieras llevarte al trabajo.
}

- Tener la capacidad de llegar a grandes audiencias era un privilegio en manos de unos pocos, que, en consecuencia, se veian impelidos a aprovechar sus tribunas para defender las causas que consideraban más legitimadas.

Las conjunciones consecutivas (por lo que, de modo que, asi que, de ahí (que), entre otras) introducen oraciones subordinadas consecutivas y presentan las siguientes caracteristicas: (i) ocupan la primera posición de la oración que introducen, (II) están separadas de la oración anterior por una coma o punto y coma y (ii) no están separadas por un signo de puntuación de la oración que introducen.

\section{- No me encontraba bien, asi que/de modo que/por lo que me quedé en casa.}

El nexo de ahí (que) presenta dos caracteristicas especiales: (i) se construye con subjuntivo, puesto que presenta la consecuencia como un hecho ya conocido, y (II) puede construirse con una oración (con que) o con un nombre (sin que).

- No me encontraba bien, de ahi que me quedara en casa.
- No me encontraba bien, de ahi mi silencio/que estuviera callado. to, por consiguiente, en consecuencia, consiguientemente, asi pues, entre otros) presentan las siguientes caracteristicas: (i) pueden ocupar la posición inicial, intermedia o final de la oración y (II) están separados del resto de elementos de la oración mediante pausas (comas en la escritura).
Los conectores discursivos consecutivos (por ello/eso, por (lo) tan-

Imagen 14. C de C1. Ficha de gramática para complementar el tratamiento de los marcadores consecutivos. Incluye información posicional y de ámbito.

En conclusión, de los ocho manuales analizados se extrae un tratamiento de los marcadores discursivos (más o menos directo/ indirecto) de seis de ellos. En líneas generales, existe un paralelismo con los requisitos del PCIC y el MCER y las tipologías incluidas, a excepción de dos carencias claras: por un lado, el aprendizaje de los marcadores discursivos de la oralidad (sobre todo, modales e interpersonales); por otro lado, los marcadores discursivos de reformulación (idea ya explorada en Salameh, 2020). Abundan ejercicios centrados sobre todo en la comprensión lectora o la 
expresión escrita, sin trabajar tanto la comprensión y expresión oral (o sin hacerlo con contextos más realistas). Además, las nociones de posición y ámbito solo se ven reflejadas en $\mathrm{C}$ de $\mathrm{C} 1$, a pesar de que esta información sería útil en cualquier nivel de aprendizaje, y más si se establece una relación con el funcionamiento de los marcadores según la posición y el ámbito que ocupen. Estas ideas suponen el punto de partida de nuestra propuesta didáctica, presentada a continuación.

\section{Propuesta didáctica}

\subsection{Características generales de la propuesta}

Nuestra propuesta de actividades sobre marcadores discursivos tiene un enfoque comunicativo, con el fin de plasmar las cuatro destrezas (auditiva, lectora, oral y escrita) y que su estudio no sea únicamente a través de la escritura o mediante ejercicios mecánicos. La propuesta se orienta a un nivel B2.2, aunque puede tomarse como modelo para adaptarla a niveles inferiores o superiores. Dado que el estudio de los marcadores discursivos no debería ser aislado con respecto a otros contenidos del temario, lo ideal es integrarlo dentro de una temática más amplia. En este caso, se ha escogido la política como base, ya que en un nivel B2.2 los estudiantes poseen capacidad para asimilar estos temas, pueden expresarse adecuadamente hablando y por escrito al haber trabajado otros contenidos previos (sobre todo, gramaticales), y, además, muestran interés debido a la posibilidad de poder relacionarlos directamente con temas políticos de actualidad de sus países de origen.

Para acotarla adecuadamente, la propuesta se centra en el grupo de los marcadores interactivos en su mayor grado de polifuncionalidad (¿no?, ¿sabes?, ¿eh?, oye, mira, etc.), ya que observamos que una mayor ausencia en los manuales analizados, aunque se incorporarán otros marcadores discursivos aprendidos en niveles previos (conectores, estructuradores de la información, operadores, incluso marcadores de reformulación). El principal objetivo consiste en integrar los conceptos de ámbito y posición (§2), ausentes por lo general en el tratamiento de los marcadores, y que pueden ser de gran utilidad a la hora de sistematizar los rasgos de los marcadores interactivos.

\subsection{Explotación didáctica: Y tú... ¿eres políticamente correcto?}

Esta propuesta didáctica consta de tres sesiones presenciales con una duración aproximada de 50 minutos para un grupo de clases semanales; el número de sesiones coincide con la duración aproximada de una unidad en este tipo de grupos. El título de la propuesta supone el punto de partida para el desarrollo de todas las sesiones, y tiene un alto componente pragmático: se trata de que los estudiantes comprueben que hablar sobre temas políticos requiere de una interacción directa con otros hablantes (y que trabajar esto por escrito no termina de ser productivo), y que esa interacción implicará una falta de acuerdo debido a diferencias de pensamiento. Para paliar estos (des) acuerdos, el trabajo con marcadores discursivos interactivos es esencial (para atenuar, mantener el turno de habla, robar el turno a otros participantes, apelar directamente a otros interlocutores, etc.). 


\section{Sesión 1. Y tú... ¿eres políticamente correcto? (50 min.)}

La pregunta clave que, probablemente, algunos estudiantes, al principio, no entenderán: ser o no políticamente correcto. Ser políticamente correcto tiene implicaciones que van más allá de hablar de política: evitar decir cosas que pueden resultar ofensivas, en mayor o menor grado, en situaciones comunicativas diferentes. Se puede ser políticamente correcto en nuestro día a día, al hablar de ciertos temas que conducen a opiniones delicadas y que, por tanto, tal vez no gusten a todo el mundo. Hacer lo contrario; es decir, no respetar algunos límites en comunicaciones comunicativas, o no cuidar aquello que se dice, sin importar la posibilidad de afectar a los demás, es ser políticamente incorrecto. Tras hablar sobre esto (primeros 5 minutos), se pide a los estudiantes que piensen en más situaciones en las que ellos podrían ser políticamente correctos o incorrectos, y que lo razonen (10 minutos de debate abierto, sin planificación escrita previa). Tras exponerlas, en grupos de tres-cuatro estudiantes, pensarán en temas que implican ser más políticamente correctos, sobre todo en sus culturas de origen. Prepararán un documento con los temas y razones a favor o en contra para ser o no políticamente correcto (10 minutos) y, posteriormente, se expondrán en grupo al resto de la clase. Finalmente, esto permitirá al docente conducir la explicación hacia un concepto pragmático básico: la imagen pública, que puede ser positiva o negativa (Brown y Levinson, 1987), y se relacionará el concepto de corrección política con ambos tipos de imagen. En esta primera sesión, se trabajan la competencia escrita y la oral (sobre todo esta última).

\section{Sesión 2. Recursos para ser políticamente correcto (50 min.)}

Después de una primera toma de contacto con la idea central de la propuesta, y una vez encontrados algunos de los temas que siempre generan diferencias entre hablantes, los alumnos aprenderán a detectar situaciones comunicativas de este tipo en varios contextos. Se introduce a los estudiantes al formato televisivo de la tertulia política, cuyo proceso de coloquialización en español peninsular es cada vez más alto (5-10 minutos). A diferencia de lo que se espera en un discurso televisivo formal, algunos de los rasgos que encontramos en las tertulias son (Briz, 2011: 128):

a. Relación entre interlocutores más cercana: los colaboradores de las tertulias son habituales, por lo que guardan una relación ligeramente estrecha. En muchas ocasiones, a pesar de que estén en televisión, los colaboradores reflejan ese grado de confianza en su modo de comunicarse.

b. Marco de interacción más familiar: si bien es cierto que las tertulias se dan en un plató, la idea de familiaridad en televisión es más frecuente. Los espectadores ya están acostumbrados a que ese sea el espacio en el que se debaten ciertos temas de interés. Esto puede propiciar un grado de cercanía comunicativa mayor.

c. Planificación sobre la marcha: las tertulias o debates políticos tienen un grado de planificación alto, puesto que parten de un guion de programa que debe respetarse. Sin embargo, el hecho de tratar sobre temas actuales y de interés político-social provoca que exista un punto de libertad a la hora de articular las intervenciones. En estos casos, puede existir una menor planificación en la elaboración de respuestas y opiniones.

d. Fin interpersonal: las tertulias políticas no tienen un fin interpersonal puro (es decir, no buscan estrechar lazos entre colaboradores), pero sí intentan conectar 
con un público que cada vez recibe mejor un grado de proximidad con el espectador en diferentes formatos televisivos (programas de realidad, programas nocturnos, franja matinal, etc.) (López Serena, 2014). Por esta razón, se podría pensar que el fin interpersonal existe, aunque sea por un beneficio propio (conseguir más audiencia).

e. Tono informal: por todo esto, en numerosas ocasiones se desprende un tono informal de las tertulias políticas (además del propio lenguaje, no se respetan los turnos de habla, los colaboradores se solapan sin escuchar al resto, etc.).

Por todo ello, será posible encontrar puntos de conflicto comunicativo y, por tanto, analizar los recursos lingüísticos utilizados en esos casos; asimismo, también podrá apreciarse cuando los interlocutores sí intentan respetar las reglas comunicativas y, por tanto, emplean recursos para ser políticamente correctos (suavizar sus ideas, buscar un punto de entendimiento común con los demás, etc.). Son modelos de uso real del lenguaje, y su análisis es significativo.

El programa de tertulia televisiva política seleccionado es LaSexta Noche, un programa de actualidad y entrevistas de corte crítico que ahondan en varios temas de interés para el público (social, político y económico), y que está en emisión desde $2013^{6}$. Normalmente, los colaboradores invitados exponen abiertamente ideologías políticas bastante distantes (siempre hay una división clara entre izquierda y derecha política). Esto genera mucha disparidad de opiniones y, por tanto, interés entre el público.

Para esta segunda sesión, los alumnos se juntarán, idealmente, en cuatro grupos de cuatro-cinco estudiantes. El profesor hará una selección de cuatro fragmentos del programa en los que se den las condiciones analizadas anteriormente. La duración de los fragmentos será, aproximadamente, de cuatro minutos. Estos fragmentos contendrán una transcripción de contenidos para facilitar la comprensión durante su visualización. La selección de los fragmentos no es azarosa: debe contener marcadores discursivos para trabajar sus usos más orales, en línea con las ideas argumentadas en este artículo. Se pedirá a los estudiantes que lleven a esa sesión un dispositivo que les permita visualizar el fragmento de manera autónoma (15 minutos). Un par de ejemplos de fragmentos que podrían trabajar los alumnos son los siguientes (puede observarse la presencia de varios marcadores del discurso en ellos):

1) A: (...) entonces, no puede ser, Toni, tienes que dejar la política, y hacerte de izquierdas, y entonces como si te hacen una oficina, mira. Los que critican esto son aquellos que no les parece un escándalo (...)

B: (intenta hablar, pero no puede)

2) A: Ya la Secretaría general me parece un escándalo, ¿no? Porque pones una Dirección General y ya está, no, no. Aquí, para colocar a un amiguete, ¿no? un amiguete, pues se crea un ministerio (...)

Tabla 2. Ejemplos transcritos del programa de tertulia politica LaSexta Noche.

\footnotetext{
${ }^{6}$ Se puede acceder al programa desde este enlace: https:/www.lasexta.com/programas/sexta-noche/
} 
Tras aclarar dudas sobre el vocabulario de los fragmentos por cada grupo (5 minutos), se pondrá el foco en el tipo de intervenciones de los hablantes $\mathrm{y}$, sobre todo, el uso de los marcadores en estos contextos comunicativos (30 minutos). El colaborador del programa ha utilizado, en esta muestra, varios marcadores interactivos (controladores del contacto, como mira, y apoyos a la formulación, como ¿no?). A partir de estos usos reales, se analizará el funcionamiento de los marcadores y, sobre todo, qué lugar ocupan en el discurso, y su relación con el resto del contenido (es decir, posición y ámbito, respectivamente). Para ello, nos apoyaremos en la siguiente ficha, adaptada para ¿no?, pero aplicable a mira y otros marcadores utilizados, y que permitirá extraer sus características a través de la reflexión metalingüística ${ }^{7}$ :

Marcador discursivo analizado: ¿no?

a) ¿En qué situación se utiliza?

- El hablante busca que otros hablantes interactúen con él;

- El hablante suaviza el contenido de su mensaje;

- El hablante intenta encontrar la formulación adecuada para su discurso o, simplemente, hace una pausa oral;

- Otro uso del marcador que no esté aquí

b) ¿En qué posición aparece?

- Al final de la oración;

- Al principio de la oración;

- En medio del fragmento

c) ¿Con qué contenido se relaciona?

- Con la oración anterior;

- Con la oración posterior;

- Con ambas simultáneamente;

- Con algo que ha dicho otro hablante;

Tabla 3. Ficha modelo para analizar la posición y el ámbito de los marcadores de manera adaptada, y vinculando ambos conceptos con el funcionamiento del marcador.

El objetivo es que, a partir de esta reflexión, los estudiantes entiendan que los marcadores discursivos interactivos muestran unos patrones de uso que pueden interiorizarse, y que son patrones precisamente porque la posición y el ámbito permiten una sistematización. La tarea de análisis se hará para los diferentes marcadores de cada grupo. Se evita, en todo momento, brindarles previamente una lista de funciones de los marcadores analizados, con el fin de que sean ellos quienes saquen características de funcionamiento generales a partir de la ficha, que incluye todas las funciones de los marcadores que se trabajan en la sesión, sin asociarlas todavía a una forma concreta.

\footnotetext{
${ }^{7}$ En la ficha descrita, el apartado a) se corresponde con la polifuncionalidad; el b), con la variable posición; el c), con ámbito. Esta información no se añade a la ficha porque se trata del material adaptado para los estudiantes para utilizarlo en la actividad.
} 
De este modo, para la muestra anterior se llega a la idea de que el marcador ¿no? en español tiene varias funciones, pero que una de ellas es ser un apoyo formulativo, un recurso para seguir emitiendo un discurso y, de paso, mantener el turno de habla, y que, cuando tiene esta función, ¿no? se suele utilizar en una posición final de la oración que acaba de emitirse, pero que se proyecta ya hacia el siguiente contenido (por eso, permite conservar el turno de habla, por lo general).

\section{Sesión 3. Una tertulia política (50 min.)}

La tercera sesión pretende funcionar, a su vez, como tarea final. Los alumnos, por grupos, realizarán su propia tertulia política. El objetivo de esta actividad será poner en práctica los valores de los marcadores aprendidos en la sesión anterior. El profesor tendrá el rol de moderador, y podrá seleccionar el contexto y los temas políticos que formarán parte de la tertulia. Para aprovechar los materiales de la sesión anterior, se seleccionará alguno de los temas ya debatido por los colaboradores de los fragmentos analizados (5 minutos).

Seguidamente, los estudiantes prepararán, por grupos (los mismos de la sesión anterior), 5 tarjetas con preguntas y opiniones sobre el tema (pueden utilizar información complementaria de periódicos, vídeos, noticias en redes sociales, etc.). Para crear más expectación y dinamismo en la interacción, el moderador indicará a los grupos, sin que los otros lo escuchen, qué postura adoptarán (más moderada, más radical, más directa, más dialogante, etc.). En este punto, podrán emplear las ideas trabajadas acerca del concepto de corrección o incorrección política (primera sesión). La condición de la actividad es que intenten utilizar, en la medida en que el contexto lo permita, alguno de los marcadores de la sesión previa.

Una vez los grupos tengan preparadas las tarjetas y claro su rol, comenzará la tertulia. El moderador habrá visto previamente las tarjetas y lanzará temas en la pizarra, relacionados con el principal, para que los estudiantes los identifiquen y empiecen a hablar. Se dará el turno a un grupo, que iniciará el debate, y los demás tendrán que interactuar en función del rol que se les ha asignado previamente. Habrá límites de tiempo si los grupos tienden a hablar solos, sin interactuar entre ellos. Tienen permiso para hablar cuando se pueda, intentar robar el turno de habla, etc. El grupo que mejor se ajuste a su rol, y que haya utilizado adecuadamente las funciones de los marcadores con diferentes posiciones y ámbitos (el profesor medirá esto), ganará la tertulia. Es esperable que los marcadores se empleen para formular ideas, introducir opiniones más duras, marcar un desacuerdo, o llamar la atención de otros participantes del debate.

\section{Conclusiones}

Esta propuesta de actividades profundiza en un problema teórico-práctico: la enseñanza de los marcadores discursivos en segundas lenguas. Habitualmente, los profesores de E/LE conocen las dificultades que implica el aprendizaje de estos elementos para los estudiantes, normalmente por su alto grado de polifuncionalidad, o porque, en muchas ocasiones, los materiales solo permiten trabajarlos desde un enfoque más formal y escrito. Tras una revisión de algunos manuales, y detectar algunos problemas mínimos, las actividades presentadas reflejan que: (a) es posible trabajar los marcadores en el aula sin recurrir a ejercicios de huecos, listas, o únicamente procesos de escritura y lectura; (b) trabajar con conversación coloquial (o contextos cercanos a ella, como las tertulias 
televisivas y otros géneros periodísticos) permite utilizar significativamente marcadores discursivos de la oralidad; (c) los alumnos son capaces de trabajar con estos usos de los marcadores desde niveles anteriores incluso al de la propuesta.

\section{Bibliografía}

BAZZANELlA, Carla. 1986. «I connettivi di correzione nel parlato: Usi metatestuali e fatici», En K. Lichem, E. Mara y S. Knaller (ed.), Parallela 2. Aspetti della sintassi dell'italiano contemporaneo. Gunter Narr Verlag, 35-45.

Briz, Antonio, José Portolés y Salvador POnS. 2008. Diccionario de partículas discursivas del español. Disponible en: http://www.dpde.es

BRIZ, Antonio y Salvador PONS. 2010. «Unidades, marcadores discursivos y posición», En Ó. Loureda y E. Acín (ed.), Los estudios sobre marcadores discursivos en español, hoy. Madrid, Arco-Libros, 257-288.

BRIZ, Antonio. 2010. «Lo coloquial y lo formal, el eje de la variedad lingüística», En R. M. Castañer Martín, V. Lagüéns Gracia (coord.), De moneda nunca usada: Estudios dedicados a José M. ${ }^{a}$ Enguita Utrilla, 125-133.

Brown, Penelope y Stephen Levinson. 1987. Politeness. Some universals in language usage, Cambridge: Cambridge University Press.

CORPAs, Jaime, GARCíA, Eva y GARmendia, Agustín. 2013. Aula Internacional 1. Curso de español, nueva edición A1. Barcelona: Difusión.

Corpas, Jaime, GarcíA, Eva y GARMendiA, Agustín. 2013. Aula Internacional 2. Curso de español, nueva edición A2. Barcelona: Difusión.

Corpas, Jaime, García, Eva y GARMEndia, Agustín. 2013. Aula Internacional 3. Curso de español, nueva edición B1. Barcelona: Difusión.

CORPAS, Jaime, GARcíA, Eva y GARMEndiA, Agustín. 2013. Aula Internacional 4. Curso de español, nueva edición B2.1. Barcelona: Difusión.

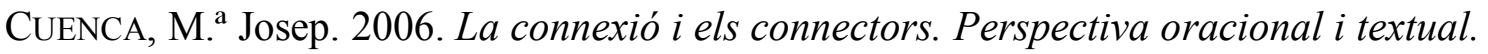
Vic, Eumo editorial Universitat de Vic.

DEGAND, Liesbeth. 2014. 'So very fast then' Discourse Markers at Left and Right Periphery in Spoken French. En Beeching, K. y U. Detges (eds.), Discourse Functions at the Left and Right Periphery, Studies in Pragmatics, 12. Leiden: Brill, 151-178.

DuQue, Eladio, DE LeÓn, Carmen Martín, y Cristina GARCía Hermoso. 2019. Palabras clave para organizar textos en español: recursos pragmáticos y discursivos. Londres: Routledge.

FISCHER, Kerstin. 2006. Approaches to discourse particles. Ámsterdam: Elsevier.

Fraser, Bruce. 1999. «What are discourse markers?», Journal of Pragmatics, 31, 7, 931952.

FRASER, Bruce. 2015. «Combinations of discourse markers in English», International Review of Pragmatics, 5, 2, 318-340.

FRETHEIM, Thorstein. 2000. «Procedural encoding of propositional attitude in 
Norwegian conditional clauses». En G. Andersen y T. Fretheim (eds.), Pragmatic markers and propositional attitude. Ámsterdam: John Benjamins, 53-84.

FUENTES, Catalina. 2009. Diccionario de conectores y operadores del español. Madrid: Arco/Libros.

HANSEN, Majj-Britt. 2008. Particles at the Semantic-Pragmatics interface: Synchronic and Diachronic issues. Amsterdam: Elsevier.

Holgado Lage, Anais. 2017. Diccionario de marcadores discursivos para estudiantes de español como segunda lengua. Berna: Peter Lang.

Instituto Cervantes. 2006. Plan Curricular del Instituto Cervantes. Madrid: Biblioteca Nueva. Recuperado de https://cvc.cervantes.es/Ensenanza/Biblioteca_Ele/plan_curricular/default.htm

JUCKER, Andreas. 1993. «The discourse marker well: A relevance-theoretical account». Journal of Pragmatics, 19, 435-452.

LÓPEZ SERENA, Araceli. 2014. «De la oralidad fingida a la oralidad simuladora de realidad. Reflexiones en torno a la coloquialización del discurso como estrategia mediática», Español Actual: revista del español vivo, 102, 35-75.

LOUREDA, Óscar y Esperanza AcíN. 2010. Los estudios sobre marcadores del discurso en español, hoy. Madrid: Arco Libros.

MARTÍNEZ SÁNCHEZ, Roser. 2011. Conectores textuales argumentativos: Guía y actividades didácticas para su uso eficaz en ELE. Barcelona: Octaedro.

MARTín Zorraquino, M. a Antonia y José Portolés. 1999. «Los marcadores del discurso», En V. Demonte y I. Bosque (eds.), Gramática Descriptiva de la Lengua Española. Madrid: Espasa-Calpe, 4051-4214.

MontañEz Mesas, Marta Pilar. 2015. Marcadores discursivos conversacionales y posición final. Hacia una caracterización discursiva de sus funciones en unidades de habla. Tesis doctoral. València: Universitat de València.

MUÑOZ PÉREZ, Julián. 2020. «El currículo discursivo en los manuales de ELE: análisis de contenidos de cuatro series de los niveles B1 y B2». Círculo de Linguística Aplicada a la Comunicación, 83, 147-168.

PORTOLÉS, José. 1993. «La distinción entre conectores y otros marcadores del discurso en español», Verba, 20, 141-170.

PORTOLÉS, José. 2001. Marcadores del discurso. Barcelona: Ariel.

Pons, Salvador. 2018. "The combination of discourse markers in spontaneous conversations. Keys to untying a Gnordian knot», En M. Borreguero, V. Atayan y S. Grosse (eds.), Models of Discourse Units in Romance Languages. Revue Romane, 53, 1, 121-158.

SALAMEH JiMÉNEZ, Shima. 2021. «Los marcadores discursivos desde una perspectiva construccional en la conversación coloquial: integración del modelo de segmentación discursiva Val.Es.Co.», En M. Sanz (coord..), Estrategias lingüísticas para la sociedad multilingüe. Barcelona: Octaedro.

SALAMEH JiMÉNEZ, Shima. 2020. «Los límites de la reformulación en la clase de E/LE», 
Revista de Profesores Foro E/LE, 16, 343-352.

SAlameH JimÉnez, Shima, Estellés, María y Salvador Pons. 2018. «Beyond the notion of periphery», En K. Beeching, C. Ghezzi y P. Molinelli (ed.), Positioning the Self and the Others: Linguistic Perspectives. John Benjamins Publishing Company, 105-125.

SCHIFFRIN, Deborah. 2015. «Discourse markers: Language, meaning and context», En D. Schiffrin, D. Tannen y H.E. Hamilton (ed.), The Handbook of discourse analysis. Oxford: Blackwell.

SCHOUROUP, Lawrence. 1999. «Discourse markers», Lingua, 107, 227-265.

TANGHE, Sanne. 2016. Marcadores derivados de los verbos de movimiento. Una aproximación cognitiva a su polifuncionalidad. Beihefte zur Zeitschrift für romanische Philologie, 408. Berlin: De Gruyer.

TraugOTT, Elisabeth-Closs. 2014. «On the function of the Epistemic Adverbs Surely and No Doubt at Left and Right Peripheries of the Clause». En Beeching, K. y U. Detges (eds.), Discourse Functions at the Left and Right Periphery, Studies in Pragmatics, 12. Leiden: Brill, 72-91.

VV.AA. 2010. Abanico. Curso de español. Nivel B2. Barcelona: Difusión. 